\title{
A Comparison of Selected Phytochemical and Antioxidant Potential of Two Tea Beverages
}

\author{
Shantrell Willis, Martha Verghese, Meijah McCollum, Kabreshia Cheatom, Zaquavis Willis, \\ Kevon Seay, Rajitha Sunkara, Lloyd Walker
}

Department of Food and Animal Sciences, Alabama Agricultural and Mechanical University, Normal, Al, USA

Email: martha.verghese@aamu.edu

How to cite this paper: Willis, S., Verghese, M., McCollum, M., Cheatom, K., Willis, Z., Seay, K., Sunkara, R. and Walker, L. (2017) A Comparison of Selected Phytochemical and Antioxidant Potential of Two Tea Beverages. Food and Nutrition Sciences, 8, 1039-1049.

https://doi.org/10.4236/fns.2017.811076

Received: September 15, 2017

Accepted: November 27, 2017

Published: November 30, 2017

Copyright $\odot 2017$ by authors and Scientific Research Publishing Inc. This work is licensed under the Creative Commons Attribution International License (CC BY 4.0).

http://creativecommons.org/licenses/by/4.0/

\begin{abstract}
Herbal teas have been used as medicinal aids for centuries to treat a myriad of illnesses and have also been suggested to function in chemoprevention. Having numerous phytochemicals, the purported health benefits of herbal teas may be due to their antioxidant potential. Herbal tea blends infused with spices are increasing in popularity in U.S., and preliminary studies suggest the synergistic effects of combining herbal teas and spices. Some teas that are increasing in popularity include raspberry leaf, strawberry leaf, and hibiscus. The objectives of this study were to determine the antioxidant capacity of tea beverages (herbal (HT) (functional yogurt-based beverage-new product) and Chai (CT) (store bought) and to determine consumer preferences of tea beverages (HT and $\mathrm{CT}$ ). Total phenolic content (TPC), total flavonoid content (TFC), free radical scavenging activity by 1,1-diphenyl-2-picryhydrazyl (DPPH), trolox equivalent antioxidant capacity (TEAC) and ferric reducing antioxidant power (FRAP) were evaluated in methanolic and aqueous beverage extracts. A functional yogurt-based beverage (HT) was developed and sensory analysis was also conducted using a 5-point hedonic scale comparing consumer preference of HT or CT beverages. The highest phenolic content was observed in CT aqueous extracts (AQ) (500 mg GAE/g), all other extracts had significantly $(P \leq 0.05)$ lower phenolic content. CTAQ had significantly $(P \leq 0.05)$ higher flavonoid content compared to all other extracts $(1.7 \mathrm{mg}$ $\mathrm{CAE} / \mathrm{g})$. The highest DPPH radical scavenging activity was observed in HT methanolic extracts (ME) (44.48\% inhibition). The lowest DPPH radical scavenging was observed in HTAQ (21.88\% inhibition). Approximately $61 \%$ of sensory panelists gave the yogurt-based developed product a rank of 4 or greater (on a 5-point hedonic scale) with $41 \%$ of panelists preferring the HT beverage over CT beverage. The results of this study suggest that a combination of herbal teas and spices may benefit consumers by improving their antioxidant status and may have potential as a functional-beverage.
\end{abstract}




\section{Keywords}

Antioxidant, Functional Food, Beverage, Herbal Tea, Sensory

\section{Introduction}

Traditional and herbal teas have been consumed for centuries, not only for their taste, but as disease preventing agents. Traditional tea, derived from the plant Camellia senesis, is the most widely consumed beverage in the world next to water [1] and market research also suggests that herbal teas are increasing in popularity in recent years. Derived from plants other than the Camellia senesis, herbal teas have been utilized for centuries for their medicinal properties. Studies suggest a positive correlation between herbal tea consumption and the reduction of select chronic diseases. Some health benefits that are associated with herbal teas include anti-cancer, hypotensive, anti-diabetic, and anti-mutagenic properties; which have been attributed to the presence of antioxidative phytochemicals [2] [3]. In recent years, the market has experienced an increase in purchases of tea and beverages containing tea (ex. Smoothies, juices). The ready to drink sector is the largest of soft drink production [4]. With less than $1 \%$ of the total food and drink market accounting for functional foods [5], consumers are continually searching for functional beverages with added benefit.

The consumption of smoothies and other yogurt based products is also on the rise in the U.S. With a $40 \%$ production increase within one year [6], yogurt contains probiotics and bioactive antioxidants from dairy; both of which can have beneficial effects on the consumer [7] [8]. Research suggests the synergistic effects of probiotics and antioxidants in the prevention or elimination of some chronic diseases [9].

Though there are few yogurt-based tea beverages on the market currently, there is still a need for more herbal tea based beverages and herbal tea blends to provide higher amounts of antioxidative phytochemicals to consumers. Some herbal teas that are increasing in popularity in the U.S. are raspberry leaf, strawberry leaf, and hibiscus teas. Tea blends are also often infused with spices for improved taste and increased positive effects on health, including the prevention of some chronic diseases such as cancer. Cinnamon, fenugreek, fennel seed and cardamom, are select spices that have been associated with health benefits but are under-utilized in the U.S.

Studies suggest the health benefits of strawberry leaf may be correlated with the high antioxidant capacity of the plant's extracts. Some reported health benefits of strawberry leaves include, anti-diarrhea, vasodilator, and antimicrobial effects [10]. Studies conducted have suggested the health benefits of raspberry leaves including, but not limited to anti-inflammatory, antiseptic, anti-diarrheic, and gastrointestinal relief [11]. Also known as sorrel, bissap, or sour tea, hibiscus 
is one of the more common herbal teas incorporated into tea blends in the U.S. [12]. A number of research efforts suggest the many health promoting properties of hibiscus including anti-hypertensive [12], hypo-cholesterolemic [13], antimicrobial and anti-cancer [14], effects.

Epidemiological studies suggest a reduction in the risk of chronic diseases, such as colon cancer, in populations with high consumption of plant foods containing phytochemicals [15]. The chemical components found in raspberry leaf (ellagic acid), strawberry leaf (quercitin), hibiscus (coumarin), cinnamon (cinnamic acid), fenugreek (trigonelline), fennel seed (rutin), and cardamom (eugenol) are reported to have anti-diabetic and anti-inflammatory potential; however, there are few ready-to-drink RTD beverages on the market that are comprised of these teas and spices. The consumption of RTD tea beverages has increased with the global tea market being almost 400 billion liters, it is estimated that RTD tea beverages will continue to grow [4].

The use of supplements and RTD tea beverages is constantly increasing. Because of this, there is a possible niche in the market for more beverages that incorporate spices and herbal teas. Though there are few yogurt-based tea beverages on the market currently, there is still a need to develop more beverages of the like and to evaluate the antioxidant capacity of the beverages to correlate the possible health benefits to consumers.

The objectives of this study were to determine the antioxidant capacity of tea beverages (herbal (HT) (functional yogurt-based beverage-new product named Zalamero) and Chai (CT) (store bought))) by evaluating total phenolic content (TPC), total flavonoid content (TFC), DPPH radical scavenging, ferric reducing antioxidant power (FRAP), and trolox equivalent antioxidant capacity (TEAC) and to determine consumer preferences of $\mathrm{HT}$ and $\mathrm{CT}$ via sensory analysis.

\section{Materials and Methods}

\subsection{Acquisition of Experimental Materials}

All chemicals were obtained from Sigma Chemical Company, St Louis, Mo. and Fisher Scientific Company, Waltham, Mass. Raspberry leaf, strawberry leaf, and hibiscus leaf teas were purchased from Monterey Bay Spice Company (Watsonville, CA). Fenugreek, cinnamon, fennel, and cardamom were purchased from a local food market. Chai tea beverage was obtained from a local market.

\subsection{Development of Beverages}

Chemical and sensorial analysis was conducted on the HT and CT beverages. The HT beverage was developed on the campus of Alabama Agricultural and Mechanical University. The formulation (Table 1) comprises of strawberry leaf, raspberry leaf, and hibiscus teas, and spices fennel seed, fenugreek, cardamom, cinnamon, yogurt, inulin, agave and frozen strawberries.

The CT beverage was obtained from a local market and contained green tea extract and soy protein isolate among other ingredients. 
Table 1. Ingredient formulation for herbal tea (HT) beverage.

\begin{tabular}{cc}
\hline Ingredient & Percent Added \\
\hline Strawberry Leaf Tea & 6.43 \\
Raspberry Leaf Tea & 6.43 \\
Hibiscus Tea & 6.43 \\
Fenugreek & 0.4 \\
Cardamom & 0.2 \\
Cinnamon & 0.15 \\
Fennel Seed & 0.2 \\
Inulin & 5.36 \\
Soy Milk & 0.85 \\
Agave & 14 \\
Yogurt & 32.38 \\
Strawberry (Frozen) & 26.59 \\
& 100 \\
\hline
\end{tabular}

\subsection{Determination of Phytochemicals in Tea Beverages}

\section{Sample preparation and extraction of phenolics:}

Extraction of HT and CT beverages was performed using established methods. Both tea beverages were dehydrated by lyophilization prior to extraction. Five grams of freeze-dried beverage powder was added to $50 \mathrm{~mL}$ of water or $80 \%$ methanol. The mixture was stirred for $2 \mathrm{hr}$ on an orbital shaker then centrifuged at $3000 \times \mathrm{g}$ for $20 \mathrm{mins}$. The supernatant was collected, filtered, and evaporated to dryness. The extraction was reconstituted with solvent and stored at $-80^{\circ} \mathrm{C}$ until further analysis.

\subsection{Determination of Total Phenolic and Flavonoid Contents in Tea Beverages}

Total phenolics in HT and CT beverages were determined by the Folin-Ciocalteau and reported as gallic acid equivalents (GAE) method [16]. Total flavonoids in beverage extracts were determined by a colorimetric method described by Zhishen et al. (1999) [17]; Adom and Liu, (2002) [18] with modifications and reported as catechin equivalents (CAE).

\subsection{Antioxidant Potential of Herbal Tea Beverages}

Antioxidant activities of HT and CT beverages were analyzed following methods described by Brand Williams et al (1995) [19] and modified by Kim et al. (2003) [20]; Lee et al. (2003) [21] for DPPH. FRAP for HT and CT beverages was determined by the method described by Benzie and Strain (1999) [22]. Trolox equivalent antioxidant capacity (TEAC) was performed following methods of Miller and Rice-Evans (1997) [23] using the 3-ethyl-benzthiazoline-6-sulfonic 
acid (ABTS) radical.

\subsection{Sensory Evaluation}

Sensory of the HT and CT beverages was conducted in the Sensory Evaluation lab at Alabama Agricultural and Mechanical University. Each of the 43 panelists were presented with coded tea beverages and a questionnaire which they used to evaluate product attributes (color, flavor, mouthfeel, appearance) via an acceptance test and a 5-point hedonic scale ranging from 1 being "Extremely Dislike" to 5 being "Extremely Like" and to indicate which product they preferred using a preference test. The panelists were also given an expectorant cup and water for rinsing between samples. The beverage samples were kept at refrigerated temperature, until presented to panelists.

\subsection{Statistical Analysis}

Results are presented as means \pm SEM using SAS system version 9.3. ANOVA was used to determine any significant differences among the treatment groups. Significance was determined at $P \leq 0.05$. The means were separated using Tukey's Studentized Range Test.

\section{Results}

There are a variety of tea beverages currently on the market, and a number of those beverages provide information on the antioxidant potential and content on the label. Antioxidant potential and content of the functional yogurt-based herbal tea (HT) beverage product was compared to that of a similar chai tea (CT) beverage purchased from a local grocery store (CT). Figure 1 and Figure 2 show the TFC and TPC of HT and CT beverages extracted with water and methanol. The CT beverages extracted with water had significantly higher $(p \leq 0.05)$ TFC (1.81 CAE/g) and TPC (523.03 GAE/g) compared to the other tea beverages. Methanolic extractions of the herbal tea beverage had significantly $(p \leq 0.05)$ higher TFC than chai tea beverage extracted with $\mathrm{MeOH}$. There were no significant differences in TPC content among extractions of HTAQ, HTMQ and CTME.

FRAP results are displayed in Figure 3. Similar to TFC and TPC results, FRAP was significantly $(p \leq 0.05)$ higher in CTAQ extracts compared to the HTME, HTAQ and CTME extracts.

Figure 4 shows the ABTS inhibition of tea beverage extracts, compared to that of vitamin $\mathrm{E}$ analog, trolox, in the trolox equivalent antioxidant capacity assay. In this assay, the ABTS radical forms a blue-green solution. As antioxidants within the sample reduce the ABTS+ to ABTS-, the blue-green color fades. This change is monitored spectrophotometrically. CTME exhibited the highest ABTS inhibition at the lowest concentration, HTAQ showed the least effective ABTS inhibition. ABTS radical was showed to be reduced by the aqueous extracts of the chai tea beverage. Figure 1 shows the ingredients list of the chai tea 


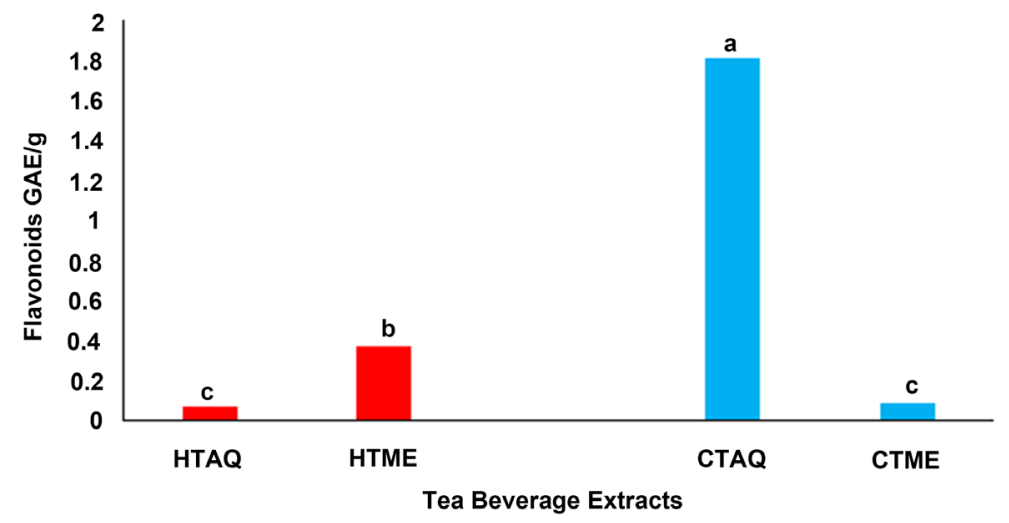

Figure 1. Total Flavonoid Content of Tea Beverage. Values are \pm SEM $\mathrm{n}=3$. ${ }^{a b c}$ Mean on bars with different letters differ $(p \leq 0.05)$ using Tukey's studentized range test. Abbreviations: $\mathrm{CAE}=$ catechin equivalents, $\mathrm{HT} \mathrm{H}_{2} \mathrm{O}=$ Herbal tea aqueous extract, $\mathrm{HT} \mathrm{MeOH}=$ Herbal tea methanolic extract, CT $\mathrm{H}_{2} \mathrm{O}=$ Chai tea aqueous extract, $\mathrm{CT} \mathrm{MeOH}=$ Chai tea methanolic extract.

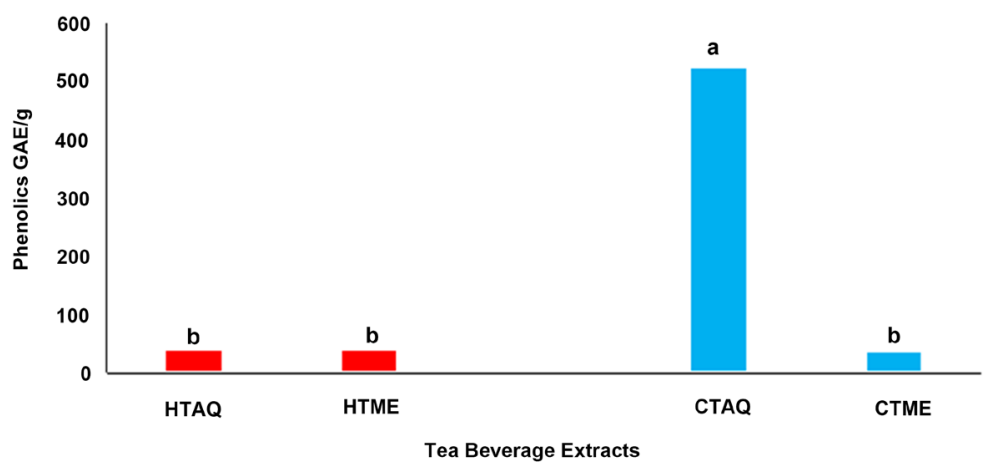

Figure 2. Total Phenolic Content of Beverages. Values are \pm SEM $n=3$. ${ }^{\mathrm{abc}}$ Mean on bars with different letters differ $(p \leq 0.05)$ using Tukey's studentized range test Abbreviations: GAE $=$ Gallic acid equivalents, HTAQ = Herbal tea aqueous extract, HTME = Herbal tea methanolic extract, $\mathrm{CTAQ}=$ Chai tea aqueous extract, $\mathrm{CTME}=$ Chai tea methanolic extract.

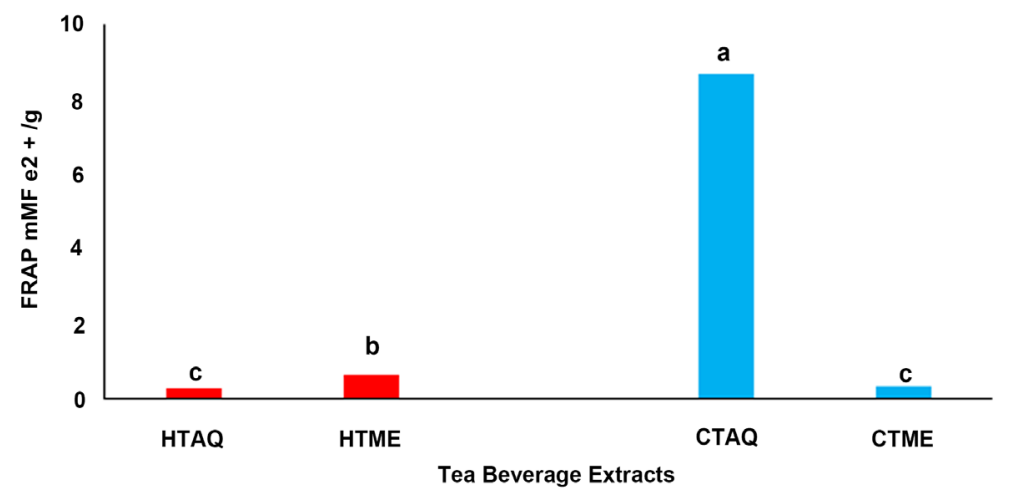

Figure 3. FRAP Value of Tea Beverages. Values are \pm SEM $n=3$. ${ }^{\text {abc }}$ Mean on bars with different letters differ $(p \leq 0.05)$ using Tukey's studentized range test. Abbreviations: FRAP $=$ Ferric reducing antioxidant power, HTAQ = Herbal tea aqueous extract, HTME = Herbal tea methanolic extract, CTAQ $=$ Chai tea aqueous extract, CTME $=$ Chai tea methanolic extract. 


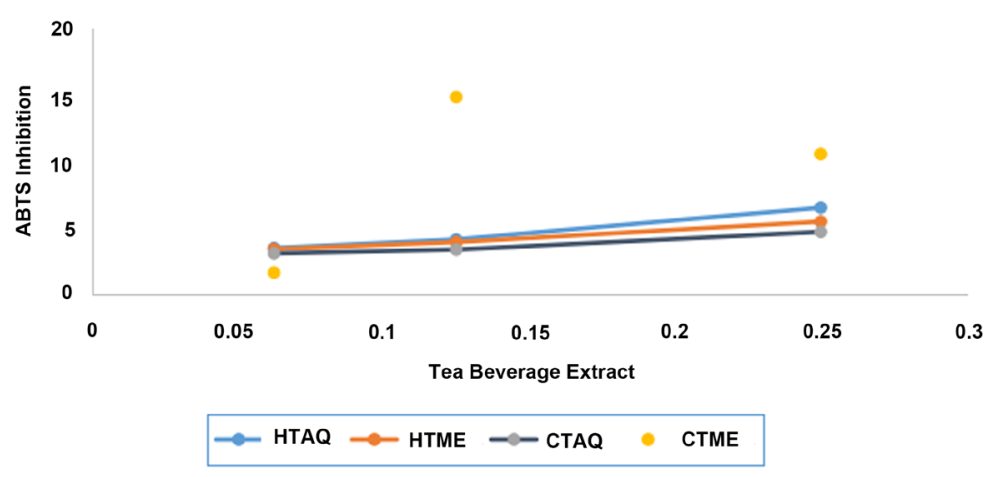

Figure 4. ABTS inhibition (TEAC) by Tea Beverages. Abbreviations: HTAQ $=$ Herbal tea aqueous extract, HTME = Herbal tea methanolic extract, CTAQ = Chai tea aqueous extract, CTME = Chai tea methanolic extract, ABTS = 2,2'azino-bis(3-ethylbenzothiazoline-6-sulphonic acid).

beverage; the beverage contained green tea extract and soy isolate, both of which have antioxidant activity.

Table 2 shows DPPH radical scavenging in HT and CT extracts. DPPH is a stable free radical that is deep purple in color. This assay measures the ability of biological samples to reduce 1,1-diphenyl-2-picryl hydrazyl radical to 1,1-diphenyl-2-picryl hydrazine; therefore a reduction in purple color indicates a reduction in free radicals. At a concentration of $0.25 \mathrm{mg} / \mathrm{ml}$ the extracts were able to inhibit the DPPH radical ranging from $21.88 \%$ inhibition (HTAQ) to $44.48 \%$ inhibition (HTME).

Sensory analysis of tea beverages was conducted on the campus of Alabama A\&M University; 43 untrained panelists were presented with herbal and chai beverages, stored at refrigerated temperature until sampling. The untrained panelists evaluated beverages using a 5-point Hedonic scale, ranging from 1 being "Dislike very much" to 5 being "Like very much". Figure 5 shows the panelists' scoring of the herbal tea beverage, with over $60 \%$ of panelists scoring the beverage a 4 or better. Figure 6 shows sensory results for the chai tea beverage, with almost $90 \%$ of panelists rating the beverage with a 4 or better. Figure 7 shows the overall preference of tea beverage. Panelists were asked to choose the beverage that they preferred, results show that $41 \%$ of panelists preferred the herbal tea beverage, while the other $59 \%$ preferred the chai beverage.

\section{Discussion}

The amount of research on smoothie and yogurt-contained beverages is steadily increasing, as interest in probiotics and their relation to disease prevention increased. Both the fruit and yogurt containing herbal tea and soy containing chai tea beverages have some levels of phenolics and flavonoid content, as according to the spectrophotometric methods that were used. The aqueous extractions of chai tea beverage had higher phenolic and flavonoid content. Soy milk is one of the prominent ingredients in the chai beverage. Soy and soy containing products have been shown to have antioxidant properties [24] some of the AOX 
Table 2. DPPH radical scavenging activity by beverage extracts.

\begin{tabular}{ccc}
\hline Tea Beverage Extract & Extract Concentration $(\mathrm{mg} / \mathrm{ml})$ & \% Inhibition \\
\hline HTAQ & 0.25 & 21.88 \\
HTME & 0.25 & 44.48 \\
CTAQ & 0.25 & 26.39 \\
CTME & 0.25 & 41.52 \\
\hline
\end{tabular}

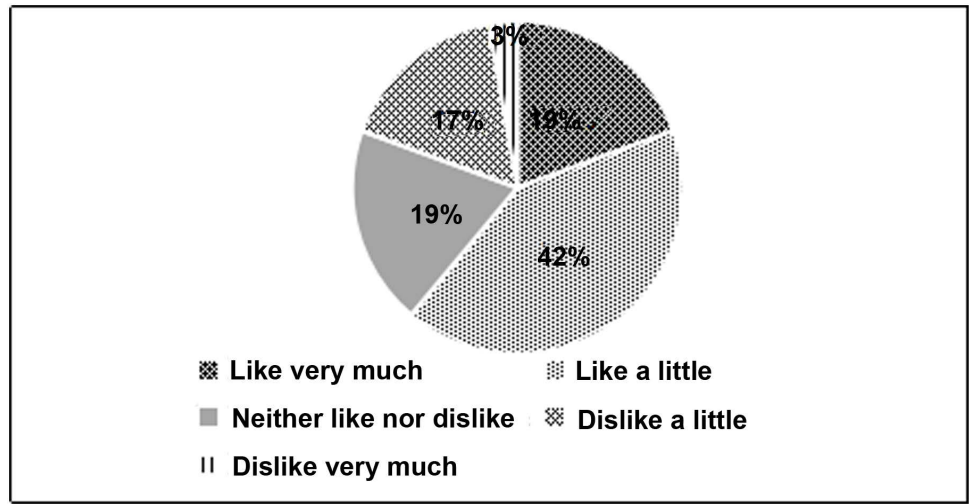

Figure 5. Acceptance of Herbal Tea (HT) Beverage by Panelists $n=43$.

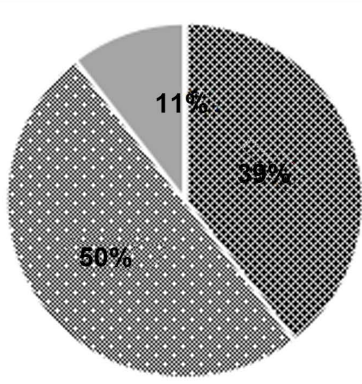

Like very much Like a little $\quad$ Neither like nor dislike

Figure 6. Acceptance of Chai Tea (CT) Beverage by Panelists $n=43$.

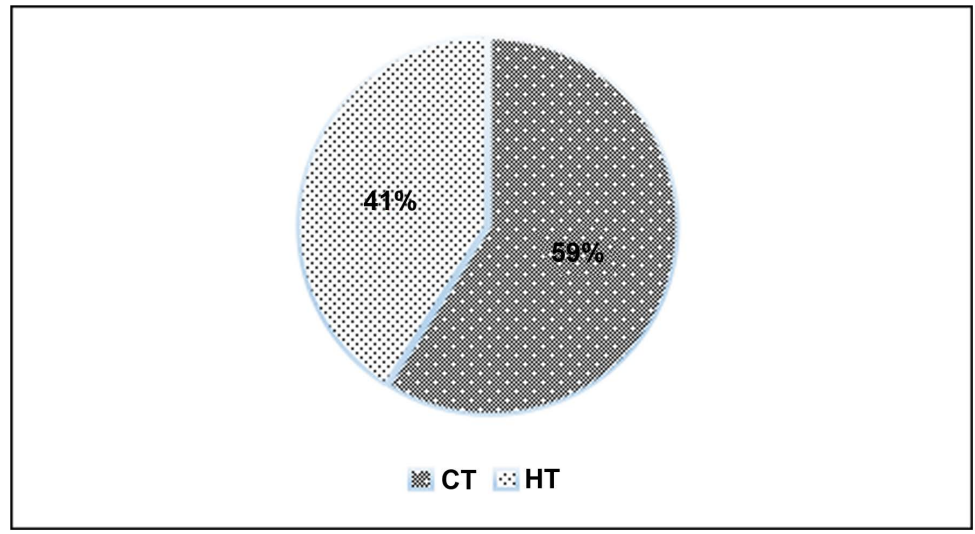

Figure 7. Overall Preference of Tea Beverages by Panelists $n=43$. 
properties are attributed to the presence of phytochemicals within soy (i.e. is oflavones). The chai tea beverage also contains green tea extract, whereas the herbal tea beverage contains strawberry leaf, raspberry leaf, and hibiscus teas. Green tea has proven to act as a powerful antioxidant with implication in multiple chronic diseases, including cancer (2 - 3). Green tea also contains a considerable amount of catechin, a flavonoid compound.

The trolox equivalent antioxidant capacity assay measures the ability of extracts to inhibition ABTS radical, compared in a water-soluble vitamin E analong, trolox. Results from this study suggest that chai tea beverage extracted with water inhibited a greater percentage of ABTS compared to other extracts. The extracts were also evaluated for DPPH radical scavenging activity, unlike TEAC results, methanolic extractions of the herbal tea beverage had more scavenging potential, compared to other extracts. This could be due to the hydrophobicity of extracts. Floefel and others compared ABTS and DPPH in assays measuring antioxidant potential in various fruits and vegetables. This study suggests that the ABTS radical is more by hydrophilic extracts [25]. Because methanol has a lesser polarity than water, a higher yield of fat-soluble compounds are anticipated to be extracted in methanolic extractions. This is one possible reason for the increased DPPH inhibition seen in methanolic extraction when compared to aqueous extractions.

ABTS radical was showed to be reduced by the aqueous extracts of the chai tea beverage. Figure 1 shows the ingredients list of the chai tea beverage; the beverage contained green tea extract and soy isolate, both of which have antioxidant activity.

The majority of the untrained panel preferred the store bought chai tea beverage over the herbal tea beverage. Though both beverages contained tea, the herbal tea functional beverage consisted of a combination of herbal teas (strawberry leaf, raspberry leaf, hibiscus) and spices (cinnamon, cardamom, fennel seed, fenugreek), some of which may not be familiar to the American palate. Some comments from panelists indicated that the herbal tea beverage was "too spicy", compared to the chai tea beverage which had a milder spice flavor. Similarly, in a study conducted by Ariefandi and others, cardamom was incorporated at various concentrations into coffee and evaluated for sensory acceptance among panelists. Though the panelists preferred the addition of cardamom at all percentages, the lowest concentration of the spice was the most preferred [26]. In a study conducted in Pakistan, fennel was used in the development of an herbal beverage; sensory results found the herbal product to be comparable to products currently on the market [27].

\section{Conclusion}

Though there are a number of functional beverages on the market currently; very few utilize herbal teas (strawberry leaf, raspberry leaf and hibiscus). The results of this study suggest that a combination of herbal teas and spices may bene- 
fit consumers by improving their antioxidant status and may have potential as a functional-beverage.

\section{References}

[1] Kuroda, Y. and Hara, Y. (1999) Antimutagenic and Anticarcinogenic Activity of Tea Polyphenols. Mutation Research, 436, 69-97. https://doi.org/10.1016/S1383-5742(98)00019-2

[2] De Vos, S. and De Schrijver, R. (2003) Lipid Metabolism, Intestinal Fermentation and Mineral Absorption in Rats Consuming Black Tea. Nutrition Research, 23, 527-537. https://doi.org/10.1016/S0271-5317(02)00556-0

[3] Haslam, E. (2003) Thoughts on Thearubigins. Phytochemistry, 64, 61-73. https://doi.org/10.1016/S0031-9422(03)00355-8

[4] Renfrew, E.C. (2016) Trends in Beverage Markets. Chemistry and Technology of Soft Drinks and Fruit Juices, 15. https://doi.org/10.1002/9781118634943.ch2

[5] Granato, D., Branco, G.F., Nazzaro, F., Cruz, A.G. and Faria, J.A. (2010) Functional Foods and Nondairy Probiotic Food Development: Trends, Concepts, and Products. Comprehensive Reviews in Food Science and Food Safety, 9, 292-302. https://doi.org/10.1111/j.1541-4337.2010.00110.x

[6] USDA. https://ndb.nal.usda.gov/ndb/

[7] Gürakan, G.C. and Altay, N. (2010) Yogurt Microbiology and Biochemistry. In: Yildiz, F., Ed., Development and Manufacture of Yogurt and Other Functional Dairy Products, 97-121.

[8] Corbo, M.R., Bevilacqua, A., Petruzzi, L., Casanova, F.P. and Sinigaglia, M. (2014) Functional Beverages: The Emerging Side of Functional Foods. Comprehensive Reviews in Food Science and Food Safety, 13, 1192-1206. https://doi.org/10.1111/1541-4337.12109

[9] Chiva, M., Soriano, G., Rochat, I., Peralta, C., Rochat, F., Llovet, T., Balanzó, J., et al. (2002) Effect of Lactobacillus Johnsonii La1 and Antioxidants on Intestinal Flora and Bacterial Translocation in Rats with Experimental Cirrhosis. Journal of Hepatology, 37, 456-462. https://doi.org/10.1016/S0168-8278(02)00142-3

[10] Yamamoto, M., Nakatsuka, S., Otani, H., Kohmoto, K. and Nishimura, S. (2000) (+)- Catechin Acts as an Infection-Inhibiting Factor in Strawberry Leaf. Phytopathology, 90, 595-600. https://doi.org/10.1094/PHYTO.2000.90.6.595

[11] McKay, D.L., Chen, C.O., Saltzman, E. and Blumberg, J.B. (2010) Hibiscus sabdariffa L. Tea (tisane) Lowers Blood Pressure in Prehypertensive and Mildly Hypertensive Adults. The Journal of Nutrition, 140, 298-303. https://doi.org/10.3945/jn.109.115097

[12] Kratchanova, M., Denev, P., Ciz, M., Lojek, A. and Mihailov, A. (2010) Evaluation of Antioxidant Activity of Medicinal Plants Containing Polyphenol Compounds. Comparison of Two Extraction Systems. Acta Biochimica Polonica, 57, 229-234.

[13] Hirunpanich, V., Utaipat, A., Morales, N.P., Bunyapraphatsara, N., Sato, H., Herunsale, A. and Suthisisang, C. (2006) Hypocholesterolemic and Antioxidant Effects of Aqueous Extracts from the Dried Calyx of Hibiscus sabdariffa L. in Hypercholesterolemic Rats. Journal of Ethnopharmacology, 103, 252-260.

https://doi.org/10.1016/j.jep.2005.08.033

[14] Olaleye, M.T. (2007) Cytotoxicity and Antibacterial Activity of Methanolic Extract of Hibiscus sabdariffa. Journal of Medicinal Plants Research, 1, 9-13. 
[15] Liu, R.H. (2007) Whole Grain Phytochemicals and Health. Journal of Cereal Science, 46, 207-219. https://doi.org/10.1016/j.jcs.2007.06.010

[16] Singleton, V.L., Orthofer, R. and Lamuela-Raventós, R.M. (1999) Analysis of Total Phenols and Other Oxidation Substrates and Antioxidants by Means of Folin-Ciocalteu Reagent. In: Lester, P., Ed., Methods Enzymol, Vol. 299, Academic Press, 152-178.

[17] Zhishen, J., Mengcheng, T. and Jianming, W. (1999) The Determination of Flavonoid Contents in Mulberry and Their Scavenging Effects on Superoxide Radicals. Food Chemistry, 64, 555-559. https://doi.org/10.1016/S0308-8146(98)00102-2

[18] Adom, K.K. and Liu, R.H. (2002) Antioxidant Activity of Grains. Journal of Agriculture and Food Chemistry, 50, 6182-6187. https://doi.org/10.1021/jf0205099

[19] Brand-Williams, W., Cuvelier, M.E., and Berset, C. (1995) Use of a Free Radical Method to Evaluate Antioxidant Activity. Lebensmittel-Wissenschaft und Technologie, 28, 25-30. https://doi.org/10.1016/S0023-6438(95)80008-5

[20] Kim, D.O., Jeong, S.W. and Lee, C.Y. (2003) Antioxidant Capacity of Phenolic Phytochemicals from Various Cultivars of Plums. Food Chemistry, 81, 321-326. https://doi.org/10.1016/S0308-8146(02)00423-5

[21] Lee, K.M., Kim, Y.J., Lee, H.J. and Lee, C.Y. (2003) Cocoa Has More Phenolic Phytochemicals and a Higher Antioxidant Capacity than Teas and Red Wine. Journal of Agricultural and Food Chemistry, 51, 7292-7295. https://doi.org/10.1021/jf0344385

[22] Benzie, I.F.F. and Strain, J.J. (1999) Ferric Reducing/Antioxidant Power Assay: Direct Measure of Total Antioxidant Activity of Biological Fluids and Modified Version for Simultaneous Measurement of Total Antioxidant Power and Ascorbic Acid Concentration. In: Lester, P., Ed., Methods Enzymol, Academic Press, Vol. 299, $15-27$.

[23] Miller, N.J. and Rice-Evans, C.A. (1997) Factors Influencing the Antioxidant Activity Determined by the ABTS+ Radical Cation Assay. Free Radical Research, 26, 195-199. https://doi.org/10.3109/10715769709097799

[24] Mahn, K., Borrás, C., Knock, G.A., Taylor, P., Khan, I.Y., Sugden, D., Aaronson, P.I., et al. (2005) Dietary Soy Isoflavone Induced Increases in Antioxidant and eNOS Gene Expression Lead to Improved Endothelial Function and Reduced Blood Pressure in Vivo. The FASEB Journal, 19, 1755-1757. https://doi.org/10.1096/fj.05-4008fje

[25] Floegel, A., Kim, D.O., Chung, S.J., Koo, S.I. and Chun, O.K. (2011) Comparison of ABTS/DPPH Assays to Measure Antioxidant Capacity in Popular Antioxidant-Rich US Foods. Journal of Food Composition and Analysis, 24, 1043-1048. https://doi.org/10.1016/j.jfca.2011.01.008

[26] Ariefandi, N. and Rizki, V.M. (2015) Development of Cardamom (Amomum cardamomum) Herbal Coffee Beverages: A Study of Physicochemical Characteristic and Consumer Perception towards Sensory Properties. Pelita Perkebunan (a Coffee and Cocoa Research Journal), 31, 49-58.

[27] Akther, S., Hussain, A. and Iman, S. (2010) Preparation and Evaluation of Physicochemical Characteristics of Herbal Drink Concentrate. Pakistan Journal of Biochemistry \& Molecular Biology, 43, 149-152. 